\title{
New Discoveries and Surprises Revealed through SALT Spectroscopy of the Unclassified Fermi-LAT Sources
}

\section{B. van Soelen*广}

University of the Free State, Bloemfontein, South Africa

E-mail: vansoelenb@ufs.ac.za

\section{Klindt, J.P. Marais, R.J. Britto, P.J. Meintjes}

University of the Free State, Bloemfontein, South Africa

\section{P. Väisänen}

South African Astronomical Observatory, Cape Town, South Africa

\section{Hanlon, D. Murphy}

University College Dublin, Dublin, Ireland

\begin{abstract}
The Fermi Space Telescope has detected 1444 objects which in the Fermi-3LAC catalogue are associated with extra-galactic sources (above galactic latitudes of $|b|>10^{\circ}$ ). While most are already associated with Flat Spectrum Radio Quasars (FSRQs) and BL Lacs, a significant fraction are associated with blazar-like sources that remain unclassified. We have undertaken optical spectroscopy and photometry of a selection of these sources from South African based telescopes. Optical spectroscopy has been used to classify these sources into FSRQs and BL Lac type objects as well as establish their redshifts. Photometric observations have searched for intraday and short-term variability in these sources. The results for four sources, 2FGL J0044.7-3702, 2FGL J0201.5-6626, 2FGL J0644.2-671 and 2FGL J0730.6-6607 are presented. In addition, long-term optical monitoring of known and candidate TeV blazars is being performed with the Watcher Robotic Telescope and a new photometric pipeline is being developed to allow for automated alerts of flaring sources which may be used to trigger follow-up observations. The preliminary results from this pipeline for PKS 1510-089 during 2015 are presented.
\end{abstract}

Frontier Research in Astrophysics - II

23-28 May 2016

Mondello (Palermo), Italy

\footnotetext{
${ }^{*}$ Speaker.

${ }^{\dagger}$ This paper uses observations made at the South African Astronomical Observatory (SAAO)
} 


\section{Introduction}

Since operation began in 2008, the Large Area Telescope (LAT) on-board the Fermi Space Telescope has been continuously observing the whole sky in the $\mathrm{MeV}$ and $\mathrm{GeV}$ energy range. This is an invaluable tool for the detection of new extra-galactic gamma-ray sources and the continuous monitoring of known sources. The recent improvement in the Fermi-LAT data analysis with the development of Pass 8 has increased the system's sensitivity over the whole energy range, and has raised the high energy detection limit. This improves the ability of Fermi-LAT to identify sources both below $100 \mathrm{MeV}$ and above $50 \mathrm{GeV}$. In addition, the improvement in sensitivity at the lower energies by Imaging Air Cherenkov Telescopes (IACTs) allows an overlap to be made between these instruments (see e.g. [1] for preliminary comparison between Fermi-LAT and H.E.S.S.).

The $3^{\text {rd }}$ Catalogue of Active Galactic Nuclei Detected by the Fermi Large Area Telescope (3LAC) [2] lists all sources detected by the instrument above a Galactic latitude of $|b|>10^{\circ}$. The clean sample contains 1444 objects, which includes 414 Flat Spectrum Radio Quasars (FSRQs) 604 BL Lacs and 24 non-blazar AGNs. The catalogue also lists 402 sources which are classified as blazar candidates of uncertain type (BCUs). This provides a wealth of new objects which may be observed at $\mathrm{TeV}$ energies with ground based IACTs. Sixty of these BCUs are detected above $50 \mathrm{GeV}$ [3] which may make them excellent new candidates if they are at redshifts $z \lesssim 1$.

As part of an on-going project we are undertaking to spectroscopically classify a selection of BCUs as well as undertake long-term photometric observations of known and candidate $\mathrm{TeV}$ sources. Below we briefly discuss recent results from this project.

\section{Spectroscopic and photometric observations of 2LAC candidate sources}

Optical spectroscopic observations have thus far been undertaken of sources which were originally selected from the $2^{\text {nd }}$ Catalogue of Active Galactic Nuclei Detected by the Fermi Large Area Telescope (2LAC) [4]. These sources all remained unclassified in the updated 3LAC. The sources were selected based on being unclassified, (mainly) radio bright (>100 mJy), observable from South Africa (latitude and magnitude limitations), and having no redshift measurement. Based on these criteria we selected thirteen sources for spectroscopic and photometric observations.

\subsection{Spectroscopic observations}

Optical spectroscopy of our sample was performed with the South African Astronomical Observatory SAAO 1.9-m telescope using the grating spectrograph between 21 May 2014 and 3 June 2014. The grating spectrograph was utilized with a wavelength coverage of $3700-7900 \AA$ with a resolution of $\sim 5 \AA$. Further observations were made with the Southern African Large Telescope (SALT) [5] using the Robert Stobie Spectrograph (RSS) [6] between November 2014 and October 2015 (2014-Semester II and 2015-Semester I). SALT/RSS observations were performed with two different instrument configurations: during 2014-Semester II grating pg0300 (wavelength range $\sim 990-11000 \AA, R \sim 530$ ) was used, which covers all observable wavelengths, and during 2015Semester I grating pg0900 (wavelength range $\sim 4000-8700 \AA, R \sim 860$ ) was used. 
Data reductions followed the standard IRAF [7] procedures, and the redshifts and classifications were determined for five sources with three additional determinations based on the most likely line identification.

\subsection{SHOC Photometric observations}

Rapid photometric observations for nine sources were obtained using the Sutherland High Speed Optical Camera (SHOC) [8] on the SAAO 1.9-m telescopes during three observing periods (13 - 19 August 2014, 10 - 31 December 2014 and 13 - 26 May 2015). Observations were undertaken with the clear filter, with exposure times between $\sim 10-200$ seconds. Data reductions were performed using the SHOC data reduction pipeline ${ }^{1}$, and differential photometry was performed using at least two comparison stars in the field-of-view following a similar method to [9].

Intraday and short-term ( few days) variability was searched for by using the power-enhanced F-test $\left(F_{\text {enh }}\right)$ analysis of variance technique [10]. Sources were classified as variable if $F_{\text {enh }}>$ $F_{\alpha=0.001}$, as possibly variable if $F_{\alpha=0.01}<F_{\text {enh }}<F_{\alpha=0.001}$, and non-variable if $F_{\text {enh }}<F_{\alpha=0.01}$. Here, $\alpha$ is the critical value (see [10] and references therein).

\subsection{LAC results}

While results have been obtained for all sources, here we briefly summarize the results for four interesting sources, with the important results summarized in Table 1. The spectra for the four sources are shown in Fig. 1.

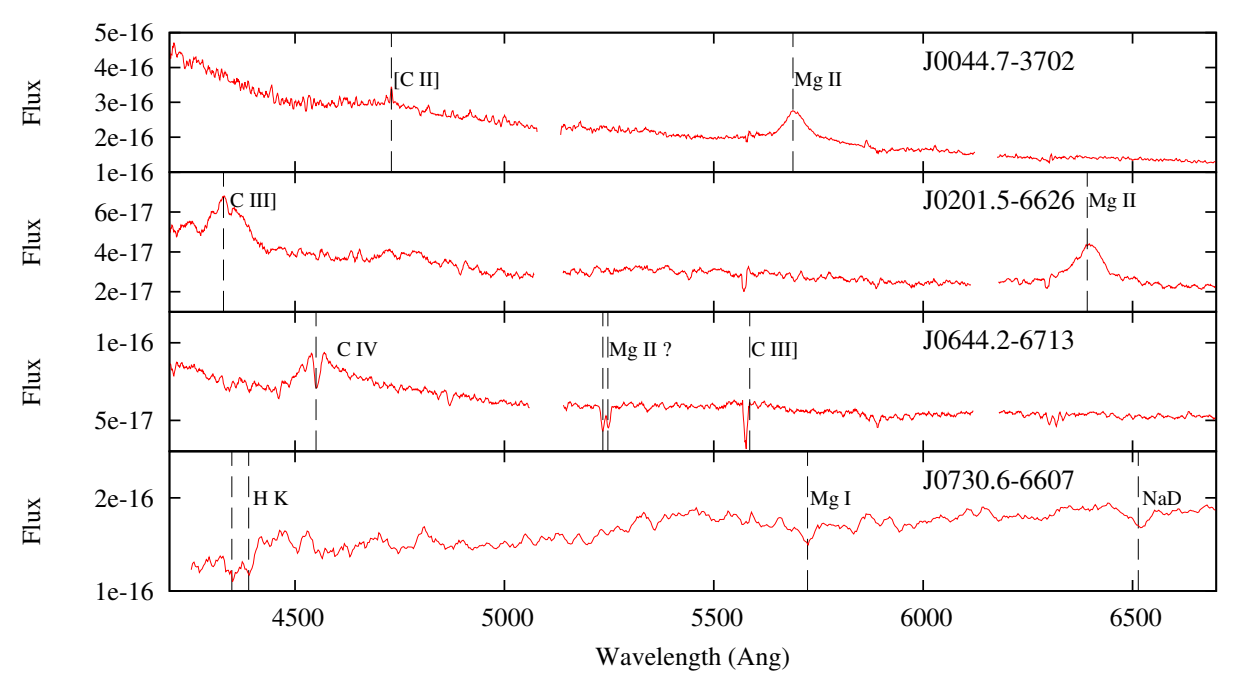

Figure 1: SALT spectra of, from top to bottom, 2FGL J0044.7-3702, 2FGL J0201.5-6626, 2FGL J0644.267132 and 2FGL J0730.6-6607. The spectra have been shape corrected but the flux is in arbitrary units. The lines used for classification and redshift determinations are indicated. Please see the text for details.

\subsubsection{FGL J0044.7-3702}

This source showed strong [C II] and Mg II emission lines which places it at a redshift of $z=1.0331 \pm 0.0004$. Based on the strength of the Mg II line, which has an equivalent width of

\footnotetext{
${ }^{1}$ The SHOC data reduction pipeline by M. Kotze can be accessed at http://shoc.saao.ac.za/Pipeline/
} 
$W_{\lambda}=17.9 \pm 3.4 \AA$, we classify it as an FSRQ. While five nights of optical observations showed no clear variability, this source has shown clear gamma-ray variability over the four year period covered by the $3 \mathrm{LAC}$, having a variability index of 173.747 [2], ${ }^{2}$ and it is a relatively bright radio source $(\sim 330 \mathrm{mJy}$ at $4.85 \mathrm{GHz})$.

\subsubsection{FGL J0201.5-6626}

This source also exhibits strong emission lines, namely $\mathrm{C}$ III] and $\mathrm{Mg}$ II, at a redshift of $z=1.28 \pm 0.01$. The equivalent widths $\left(W_{\lambda, C} I I I\right]=31 \pm 13 \AA$ and $\left.W_{\lambda, M g I I}=42 \pm 12 \AA\right)$ classify the source as an FSRQ. While gamma-ray variability was not significantly detected, 6 nights of observations showed clear optical variability.

\subsubsection{FGL J0644.2-6713}

This source exhibits broad C IV and C III] emissions lines at a redshift of $z=1.930 \pm 0.004$ (which is the highest redshift in our sample). The equivalent width $\left(W_{\lambda, C I V}=24 \pm 13 \AA\right.$ ) classifies this as an FSRQ. However, this source also exhibits two intervening absorption lines in the spectra. One lies over the broad C IV line, which suggest absorption for material further out from the galactic nucleus. The second is a double absorption feature at $\sim 5240 \AA$ which may be due to absorption from an intervening galaxy. We suggest that this is Mg II at a redshift of $z \approx 0.9$. Gamma-ray variability has been reported for this sources in the 3LAC and observations over 8 nights with SHOC showed variability on 5 nights and clear variability over the whole observing period.

\subsubsection{FGL J0730.6-6607}

The spectrum of 2FGL J0730.6-6607 is mainly featureless, which classifies this as a BL Lac, and $\mathrm{Ca}$ II $\mathrm{H} \& \mathrm{~K}, \mathrm{Mg}$ I and $\mathrm{NaD}$ absorption lines place the source at $z=0.106 \pm 0.001$. The weak $\mathrm{Ca}$ II depression is consistent with a significant non-thermal contribution to the observed spectrum. Optical photometric observations showed intraday variability over three nights and overall shortterm variability. While there was no reported gamma-ray variability in the 3LAC this is the hardest source in our sample $(\Gamma=1.34)$. Since the source lies at a relatively low redshift this is our best candidate for IACT observations at $\mathrm{TeV}$ energies.

\section{Long-term monitoring optical monitoring}

In addition to the follow-up studies on candidates $\mathrm{TeV}$ sources we are also undertaking longterm optical monitoring of known and candidate TeV blazars with the Watcher Robotic Telescope [11]. As part of this process an automated pipeline is being developed in Python to undertake rapid photometric reduction of the sources.

\footnotetext{
${ }^{2} \mathrm{~A}$ variability index $>72.44$ indicates the source has a probability of $>99 \%$ of being variable over the 4 -year timescale considered in the 3LAC.
} 
Table 1: Summary of the measured properties for four sources classified as part of this study. The table list the lines identified, the redshift, the classification based on the line profiles, the number of nights that show variability $(\mathrm{V})$, potential variability $(\mathrm{PV})$, no variability $(\mathrm{N})$ and whether short-term variability was detected (STV).

\begin{tabular}{lccccccc} 
Source & Lines & Redshift & Classification & V & PV & N & STV \\
\hline 2FGL J0044.7-3702 & [C II], Mg II & $1.0331 \pm 0.0004$ & FSRQ & 0 & 2 & 3 & no \\
2FGL J0201.5-6626 & C III], Mg II & $1.280 \pm 0.010$ & FSRQ & 2 & 0 & 4 & yes \\
2FGL J0644.2-6713 & C IV, C III] & $1.930 \pm 0.004$ & FSRQ & 5 & 1 & 2 & yes \\
2FGL J0730.6-6607 & Ca II H\&K, Mg I, NaD & $0.1063 \pm 0.0009$ & BL Lac & 3 & 2 & 1 & yes \\
\hline
\end{tabular}

\subsection{Pipeline procedure}

The photometric pipeline being developed makes use of PYRAF, an IRAF interpreter written in Python. For all data frames bias and flat field correction is first performed (the dark current is negligible), after which all frames are corrected to the same orientation. Next the SEP module [12], using the Source Extractor [13] program, determines the properties of the sources and background sky. This includes, for example, source positions, source ellipticity ${ }^{3}$ and the sky background level and standard deviation. Data frames are rejected if the sources have an ellipticity $e>0.9$, (indicating poor tracking due to e.g. wind), or if the sky background is more than twice the global average (which indicates, for example, that the target was too close to the Moon).

All accepted frames are then analysed using the IRAF tasks DAOРНОT/РНОT to perform aperture photometry on all objects in the field of view using input parameters determined from the SEP package. Differential photometry of selected sources is then performed following [9].

\subsection{Photometric monitoring of PKS 1510-089}

As an example of the output from the pipeline, Fig. 2 shows the long-term V, R and V-R light curves for PKS 1510-089 during 2015. The source shows large variability as is expected for this well known blazar and there is some indication of the colour variations.

The pipeline is still under development and we intend to improve the aperture optimization. However, this example demonstrates the ability of the Watcher telescope to undertake this longterm monitoring campaign.

\section{Discussion and Conclusion}

Thus far, through the BCU follow-up programme we have been able to classify approximately 8 new blazar sources into FSRQs and BL Lac type objects and establish their redshifts. These are crucial in searching for new potential $\mathrm{TeV}$ sources that are close enough to be observed with ground based IACTs. We have identified at least one source, namely 2FGL J0730.6-6607, which has a hard gamma-ray photon index $(\Gamma=1.34)$ and lies close enough $(z=0.106 \pm 0.001)$ to be considered for $\mathrm{TeV}$ observations. The $\mathrm{BCU}$ classification is part of an on-going programme that has now been expanded with the updated 3LAC and 2FHL catalogues, which have increased the

\footnotetext{
${ }^{3}$ Here ellipticity refers to the shape of the sources on the image.
} 


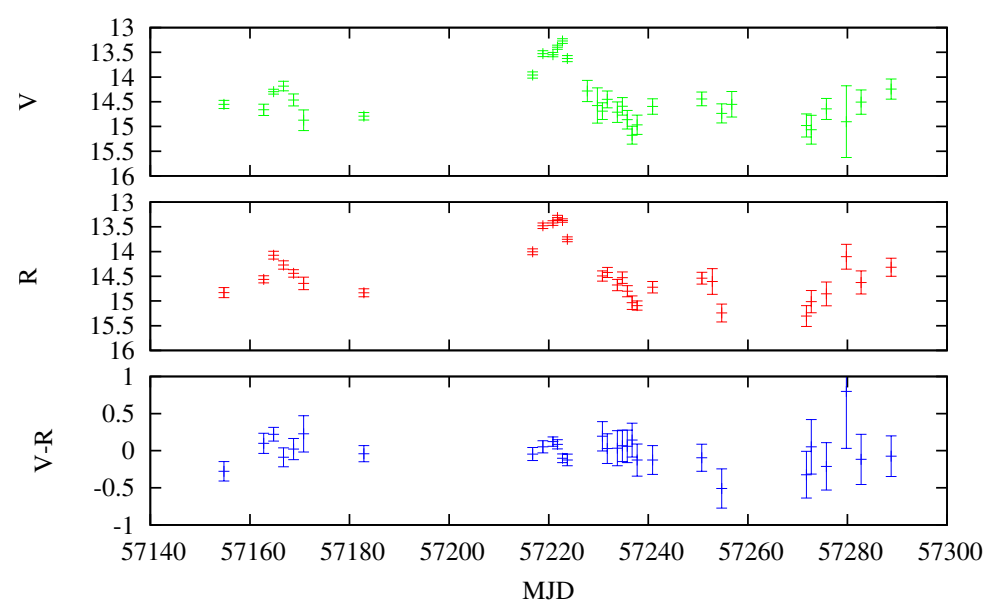

Figure 2: Long term optical light curve in V (top), R (middle) and V-R (bottom) during 2015. These data are analysed with the new data reduction and analysis pipeline.

number of BCU sources. Of particular interest is the search for rarer $\mathrm{TeV}$ candidate sources. For example, TeVCat currently ${ }^{4}$ only lists five known TeV FSRQ systems, in comparison to the more than 50 BL Lacs. A more detailed discussion of the spectroscopic observations of seven candidate sources is reported in [14].

The classification of Fermi-LAT sources is also currently being investigated using machine learning techniques $[15,16]$. Such techniques provide important methods to search for potential sources based on e.g. gamma-ray variability and photon index. However, these methods are dependent on having sources with known optical classifications and the observations discussed here can be used to improve their calibration.

In addition, the long-term optical monitoring campaign with the Watcher telescope of both the known and candidate blazar sources will allow for multi-wavelength correlations studies to be undertaken. A new pipeline is currently under development, the aim of which, is to allow for automated alerts of flaring sources which may be used to trigger follow-up observations.

The current development of the Cherenkov Telescope Array (CTA), will greatly increase the sensitivity of $\mathrm{TeV}$ observations and this on-going project aims to identify new sources that will be important candidates for future $\mathrm{TeV}$ observations.

\section{Acknowledgments}

This work is based on the research supported in part by the National Research Foundation of South Africa for the grant 87919. Some of the observations reported in this paper were obtained with the Southern African Large Telescope (SALT).

\section{References}

[1] D. Zaborov et al. AGN observations with a less than $100 \mathrm{GeV}$ threshold using H.E.S.S. II in proceedings of the 34th International Cosmic Ray Conference (ICRC2015), arXiv/1509.06509

\footnotetext{
${ }^{4}$ http://tevcat.uchicago.edu/ as of 30 July 2016
} 
[2] M. Ackermann et al. The Third Catalog of Active Galactic Nuclei Detected by the Fermi Large Area Telescope, ApJ 810 (2015) 14

[3] M. Ackermann et al. 2FHL: The Second Catalog of Hard Fermi-LAT Sources, ApJS 222 (2016) 5

[4] M. Ackermann et al. The Second Catalog of Active Galactic Nuclei Detected by the Fermi Large Area Telescope, ApJ 743 (2011) 171

[5] D.A.H. Buckley, G.P. Swart, J.G Meiring Completion and commissioning of the Southern African Large Telescope, SPIE 6267 (2006) 32

[6] E.B. Burgh et al. Prime Focus Imaging Spectrograph for the Southern African Large Telescope: optical design, SPIE 4841 (2003) 1463

[7] D. Tody The IRAF Data Reduction and Analysis System, SPIE 627 (1986) 733

[8] R. Coppejans et al. Characterizing and Commissioning the Sutherland High-Speed Optical Cameras (SHOC), PASP 125 (2013) 976

[9] M. Everett and S.B. Howell, A Technique for Ultrahigh-Precision CCD Photometry, PASP 113 (2001) 1428

[10] J.A. De Diego On the Reliability of Microvariability Tests in Quasars, AJ 148 (2014) 93

[11] M. Topinka et al. Status update of the Watcher Robotic Telescope, EAS 61 (2013) 487

[12] K. Barbary, K. Boone and C. Deil SEP: v0.3.0 URL http://dx.doi.org/10.5281/zenodo.15669 (2015)

[13] E. Bertin and S. Arnouts SExtractor: Software for source extraction, A\&AS 117 (1996) 393

[14] L. Klindt, B. van Soelen, P.J. Meintjes, P. Väisänen Optical spectroscopic classification of a selection of Southern hemisphere Fermi-LAT unclassified blazars, MNRAS 467 (2017) 2537

[15] S. Parkinson Classification and Ranking of Fermi LAT Gamma-ray Sources from the 3FGL Catalog using Machine Learning Techniques, ApJ 820 (2016) 8

[16] G. Chiaro et al. (2016) Blazar Flaring Patterns (B-FlaP): Classifying Blazar Candidates of Uncertain type in the third Fermi-LAT catalog by Artificial Neural Networks, arXiv/1607.07822 